\title{
Erratum to: Exon 19 deletion of epidermal growth factor receptor is associated with prolonged survival in brain metastases from non-small-cell lung cancer
}

\author{
Hongwei $\mathrm{Li}^{1,5}$ - Xiaqin Zhang ${ }^{1}$ Jianzhong Cao ${ }^{1}$ • Pengcheng Su${ }^{1} \cdot$ Jianhong Lian $^{2}$. \\ Xing Song ${ }^{1} \cdot$ Weihua Yang ${ }^{3}$. Songyan $\mathrm{Han}^{3}$ • Yanfeng $\mathrm{Xi}^{4}$ • Yaohua Wang ${ }^{4}$
}

Published online: 29 August 2015

(C) International Society of Oncology and BioMarkers (ISOBM) 2015

\section{Erratum to: Tumor Biol}

DOI 10.1007/s13277-015-3653-2

The original version contains mistake. The sentence "One hundred six patients with BM from NSCLC were retrospectively reviewed. Thirty-three subjects $(24.3 \%)$ were confirmed to have an exon 19 deletion, while another 33 had an exon 21 point mutation (L858R) (24.3\%)." should be "One hundred and thirty-six patients with BM from NSCLC were retrospectively reviewed. Thirty subjects $(22.1 \%)$ were confirmed to have an exon 19 deletion, while another $30 \mathrm{had}$ an exon 21 point mutation (L858R) (22.1\%)."

The online version of the original article can be found at http://dx.doi.org/ 10.1007/s13277-015-3653-2.

Hongwei Li

3420010@163.com

Jianzhong Cao

caolv2000@sina.com

1 Department of Radiation Oncology, Shanxi Provincial Cancer Hospital, Shanxi Medical University, Taiyuan, Shanxi 030013, People's Republic of China

2 Department of Surgery, Shanxi Provincial Cancer Hospital, Shanxi Medical University, Taiyuan, Shanxi 030013, People's Republic of China

3 Department of Chemotherapy, Shanxi Provincial Cancer Hospital, Shanxi Medical University, Taiyuan, Shanxi 030013, People's Republic of China

4 Department of Pathology, Shanxi Provincial Cancer Hospital, Shanxi Medical University, Taiyuan, Shanxi 030013, People's Republic of China

5 Department of Radiotherapy, Shanxi Provincial Cancer Hospital, Shanxi Medical University, Taiyuan, Shanxi 030013, People's Republic of China
The sentence "Sixty-two of the 136 BM patients were found to have EGFR mutations, including $24.3 \%$ (33) with exon 19 deletions and $24.3 \%$ (33)with exon 21 point mutation (L858R)." (Result, Patient characteristics, paragraph 1, sentence 3) should be "Sixty-two of the 136 BM patients were found to have EGFR mutations, including $22.1 \%$ (30) with exon 19 deletions and $22.1 \%$ (30) with exon 21 point mutation (L858R)."

The sentence "One hundred fifteen patients were confirmed as adenocarcinoma and 15 patients as squamous cell carcinomas." (Result, Patient characteristics, paragraph 1 sentence 6) should be "One hundred fourteen patients were confirmed as adenocarcinoma and 15 patients as squamous cell carcinomas."

The sentence "Six patients could not be discriminated between adenocarcinoma and squamous cell carcinomas." (Result,Patient characteristics, paragraph 1 sentence 7) should be "Seven patients could not be discriminated between adenocarcinoma and squamous cell carcinomas."

The sentence "The other 42 patients had received combinatorial treatment of WBRT plus systemic chemotherapy concurrently or subsequently: 18 were treated with WBRT plus EGFR-TKI, 15 were treated with EGFRTKI plus systemic chemotherapy, and 13 patients had no special treatment." (Treatment, paragraph 1 sentence 3$)$ should be "The other 43 patients had received combinatorial treatment of WBRT plus systemic chemotherapy concurrently or subsequently: 17 were treated with WBRT plus EGFR-TKI, 15 were treated with EGFRTKI plus systemic chemotherapy, and 13 patients had no special treatment."

The sentence "However, in our study, treatment method contributed to statistically significant differences in overall survival of patients with either EGFR exon 19 deletion or 21 point mutation." (Discussion, paragraph 3 sentence 4) 
should be "However, in our study, treatment method did not contributed to overall survival of patients with either EGFR exon 19 deletion or 21 point mutation."

Table 1 "ECOG PS at BM" HR value "2.033" should be "1.618".
Figure 5 Synchronic BM: "19 Exon deletions mutation $(\mathrm{n}=42)$ " should be "19 Exon deletions mutation $(\mathrm{n}=17)$ "; "no (n=17)" should be "no (n=42)". Metachronic BM: "19 Exon deletions mutation ( $\mathrm{n}=64)$ " should be "19 Exon deletions mutation ( $\mathrm{n}=13)$ "; "no $(\mathrm{n}=13)$ " should be "no $(\mathrm{n}=64)$ ". 